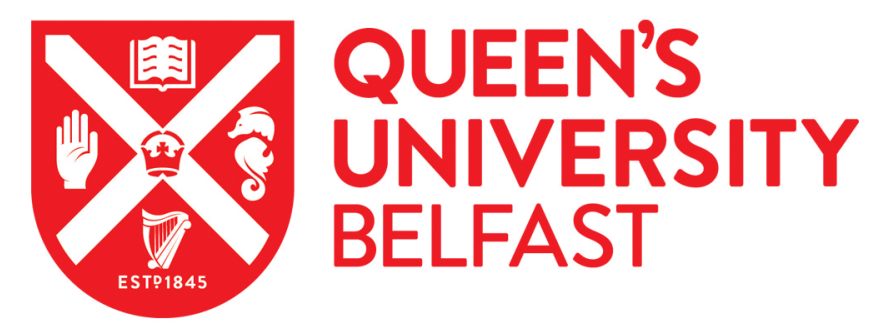

\title{
Characterizing exo-ringsystems around fast-rotating stars using the Rossiter-McLaughlin effect
}

de Mooij, E. R. J. W., Watson, C. A., \& Kenworthy, M. A. (2017). Characterizing exo-ringsystems around fastrotating stars using the Rossiter-McLaughlin effect. Monthly Notices of the Royal Astronomical Society, 472, 2713-2721. https://doi.org/10.1093/mnras/stx2142

Published in:

Monthly Notices of the Royal Astronomical Society

Document Version:

Publisher's PDF, also known as Version of record

Queen's University Belfast - Research Portal:

Link to publication record in Queen's University Belfast Research Portal

Publisher rights

Copyright 2017 Oxford University Press

This work is made available online in accordance with the publisher's policies. Please refer to any applicable terms of use of the publisher.

\section{General rights}

Copyright for the publications made accessible via the Queen's University Belfast Research Portal is retained by the author(s) and / or other copyright owners and it is a condition of accessing these publications that users recognise and abide by the legal requirements associated with these rights.

Take down policy

The Research Portal is Queen's institutional repository that provides access to Queen's research output. Every effort has been made to ensure that content in the Research Portal does not infringe any person's rights, or applicable UK laws. If you discover content in the Research Portal that you believe breaches copyright or violates any law, please contact openaccess@qub.ac.uk. 


\title{
Characterizing exo-ring systems around fast-rotating stars using the Rossiter-McLaughlin effect
}

\author{
Ernst J.W. de Mooij, ${ }^{1,2 \star}$ Christopher A. Watson ${ }^{2}$ and Matthew A. Kenworthy ${ }^{3}$ \\ ${ }^{1}$ School of Physical Sciences, Dublin City University, Glasnevin, Dublin 9, Ireland \\ ${ }^{2}$ Astrophysics Research Centre, Queen's University Belfast, Belfast BT7 INN, UK \\ ${ }^{3}$ Leiden Observatory, Leiden University, Niels Bohrweg 2, NL-2333 RA Leiden, the Netherlands
}

Accepted 2017 August 15. Received 2017 August 15; in original form 2017 June 1

\begin{abstract}
Planetary rings produce a distinct shape distortion in transit light curves. However, to accurately model such light curves the observations need to cover the entire transit, especially ingress and egress, as well as an out-of-transit baseline. Such observations can be challenging for long period planets, where the transits may last for over a day. Planetary rings will also impact the shape of absorption lines in the stellar spectrum, as the planet and rings cover different parts of the rotating star (the Rossiter-McLaughlin effect). These line-profile distortions depend on the size, structure, opacity, obliquity and sky-projected angle of the ring system. For slow-rotating stars, this mainly impacts the amplitude of the induced velocity shift; however, for fast-rotating stars the large velocity gradient across the star allows the line distortion to be resolved, enabling direct determination of the ring parameters. We demonstrate that by modelling these distortions we can recover ring system parameters (sky-projected angle, obliquity and size) using only a small part of the transit. Substructure in the rings, e.g. gaps, can be recovered if the width of the features $(\delta W)$ relative to the size of the star is similar to the intrinsic velocity resolution (set by the width of the local stellar profile, $\gamma$ ) relative to the stellar rotation velocity ( $v \sin i$, i.e. $\left.\delta W / R_{*} \gtrsim v \sin i / \gamma\right)$. This opens up a new way to study the ring systems around planets with long orbital periods, where observations of the full transit, covering the ingress and egress, are not always feasible.
\end{abstract}

Key words: techniques: spectroscopic - planets and satellites: rings.

\section{INTRODUCTION}

Within our Solar system, ring systems of varying extent are present around each of the gas- and ice-giants, the most famous being those of Saturn. Such rings are not only constrained to giant planets, however. For example, a thin dense ring has been revealed around the Centaur object Chariklo (Braga-Ribas et al. 2014), and further evidence of past ring structure around Iapetus (a satellite of Saturn) has been unveiled by the Cassini mission (Ip 2006). Beyond the confines of our Solar system, a giant ring system spanning a diameter of $\sim 0.2-0.8$ au has been discovered around an object transiting the young Sun-like star J1407 (Mamajek et al. 2012). $\beta$ Pic b may also represent another planetary system with rings that transit its host star. Its orbit is aligned closely with the line of sight (e.g. Chauvin et al. 2012; Millar-Blanchaer et al. 2015; Wang et al. 2016), and Lecavelier Des Etangs et al. (1995) found an $\sim 5$ per cent fluctuation in the light curve of this system in 1981 November. The depth of this event indicates the presence of a transit of a dust disc or ring structure surrounding the planet (Lecavelier Des Etangs \& Vidal-Madjar 2009).

It therefore seems that planetary ring systems may be relatively common throughout the Universe. Despite this, many open questions remain about the physics steering ring formation and evolution. For example, numerous theories regarding the formation of Saturn's rings have been put forward. These include the condensation model (where the rings are from the leftover remnants of a protosatellite disc-Pollack 1975), tidal or collisional disruption of a small moon (e.g. Roche 1849; Harris 1984; Charnoz et al. 2009; Charnoz 2009) or comet (Dones 1991), or that it formed from a super-massive primordial ring [for a review, see Charnoz et al. (2017).].

Another interesting aspect is the possibility of ring-satellite interactions. For instance, Saturn's ring system may have given birth to several satellites (such as Pandora and Prometheus), and the growth of these satellites may be quite rapid, over time-scales of a few Myr (Charnoz, Salmon \& Crida 2010). Larger ring systems, such as that present around J1407b, may spawn more massive moons detectable by transit surveys. Indeed, a gap within the hypothesized ring system 
of $\mathrm{J} 1407 \mathrm{~b}$ is consistent with having been cleared out by a satellite with a mass of up to $0.8 M_{\oplus}$ (Kenworthy \& Mamajek 2015).

Identifying and characterizing ring systems around exoplanets will therefore help constrain the physical processes governing the formation and evolution of rings across a wide range of ages and environments. Detection of ring gaps may also inform us of the possible presence of exomoons (some potentially habitable), providing an additional discovery path to these objects (Kenworthy 2017). Exo-rings may be observed during transit, where they produce distinct variations in the light curve (as seen in the J1407b system by SuperWASP; Mamajek et al. 2012). In addition, Ohta, Taruya \& Suto (2009) outlined how exo-rings introduce an additional velocity anomaly while observing the Rossiter-McLaughlin (RM) effect on top of the RM anomaly arising from the planet itself.

In this paper, we propose a new technique that uses all the information encoded in the stellar line profile during transit to directly characterize the ring system geometry. This has the advantage over other methods presented in the literature in that it can yield important information on transiting ring systems using only a small part of the transit, rather than requiring coverage of the full transit. This is particularly pertinent given, for example, the expected duration of some of these events. In the case of $\beta$ Pic-b, the transit of the planet/ring system is expected to take $\sim 2 \mathrm{~d}-$ making full coverage of this long event difficult. Given the predictions of upcoming transits of $\beta$ Pic-b in 2017/2018 (e.g. Lecavelier des Etangs \& VidalMadjar 2016; Wang et al. 2016), this motivated our development of this new method.

Since $\beta$ Pic is a fast rotator, the velocity gradient across the star provides an additional handle on the shape of the rings during transit. Here we present an investigation into the impact of rings on the stellar line profiles of a $\beta$ Pic-like system. In Section 2 we present our model, which we use in Section 3 to investigate the dependence of the line-profile distortions on different parameters of the ring system. In Section 4 we show (through simulations) how we can recover the parameters of the ring system, and we discuss the results in Section 5. Finally, we present our conclusions in Section 6.

\section{MODELLING THE LINE-SHAPE DISTORTIONS DUE TO TRANSITING RING SYSTEMS}

We model the integrated stellar line profile as a function of velocity, $F_{0}(v)$, using a 2D grid. We assign each pixel $[i, j]$ on the stellar surface an intrinsic line profile, $f_{i j}(v)$, taking into account the stellar rotation, characterized by $v \sin i$, assuming solid body rotation, and an intrinsic line broadening, $\gamma$, which is assumed to be the same for all locations on the star. In this paper we model the intrinsic line profile as a Gaussian:

$$
f_{i j}(v)=1-A \mathrm{e}^{-\frac{(4 \ln (2))\left(v-v_{r, i j}\right)^{2}}{\gamma^{2}}},
$$

where $A$ is the maximum strength at the centre of the line and $v_{r, i j}$ is the velocity of the centre of the line due to stellar rotation at that position.

In addition, we take limb-darkening into account using the quadratic limb-darkening law (e.g. Claret 2000). The total integrated line profile is calculated as

$F_{0}(v)=\sum_{i, j}^{\text {star }} f_{i j}(v)\left(1-u_{1}\left(1-\mu_{i j}\right)-u_{2}\left(1-\mu_{i j}\right)^{2}\right)$, where $u_{1}$ and $u_{2}$ are the linear and quadratic limb-darkening coefficients, respectively, and $\mu_{i j}$ is the cosine of the angle between the line of sight and the emission at that location.

The stellar disc was modelled using $\sim 817000$ pixels, giving a radius of 510 pixels. The disc-integrated line profile (determined using equation 2) was calculated at a velocity resolution of $250 \mathrm{~m}$ $\mathrm{s}^{-1}$ from $-250 \mathrm{~km} \mathrm{~s}^{-1}$ to $+250 \mathrm{~km} \mathrm{~s}^{-1}$. The planet was modelled as a fully opaque disc with radius $R_{\mathrm{p}}$, while the rings were modelled as concentric ellipses, centred on the planet. The rings were all assumed to be co-planar [i.e. they all have the same obliquity and position angle (PA) on the sky], and to be circular when viewed face-on. Each ring is described by three parameters, its inner radius $R_{\min , n}$, its outer radius $R_{\max , n}$ and its optical depth $\tau_{n}$ and are considered to be nested (i.e. $R_{\min , n+1} \geqq R_{\max , n}$ ). Finally, we assume the rings to be thin, such that we can specify the optical depth for each ring independent of the inclination.

To model the transit we assume a circular orbit for the planet, with an orbital period $P$, semi-major axis $a$ and an impact parameter $b$. Here we define $b$ as the minimum distance between the centre of the stellar disc and the centre of the planet's disc. For the simulations considered here we also assume spin-orbit alignment, i.e. that the orbital momentum vector of the planet's orbit is (almost) parallel to the stellar rotation axis. The close alignment of the stellar rotation and orbital axes in the Solar system (e.g. Giles 2000) is attributed to the formation of the Sun and planets from a single rotating protostellar disc that was also initially aligned perpendicular to the solarrotation axis. Studies of short period hot Jupiter systems, however, show a significant spin-orbit misalignment for stars with $T_{\text {eff }} \gtrsim$ 6250 K (e.g. Fabrycky \& Winn 2009; Mazeh et al. 2015), where it is thought that the primordial spin-orbit alignment was disrupted by the process of migration. These results may not necessarily apply to $\beta$ Pic b-like planets with orbital periods of several years. Indeed, in the case of $\beta$ Pic b itself, Currie et al. (2011) report that the planet's orbit is aligned with the flat outer debris disc. Watson et al. (2011) and Greaves et al. (2014) showed that there was no observational evidence for misalignments between stars and their debris discs, and that the general picture was one of good star-disc alignment. These studies also included some debris disc host stars with imaged planetary candidates between 15 and $180 \mathrm{au}$, which further suggested planet-disc co-planarity. Thus, the assumption of spin-orbit alignment in our models appears reasonable when considering long period planets. Furthermore, a small misalignment will have a negligible effect on the shape of the distortions.

Finally, we calculate the position of the planet as a function of time, and determine the line profile observed during transit, $F(v$, $t$ ), by subtracting the flux under the planet and rings from the full disc-integrated line profile $F_{0}(v)$ taken when the planet and rings are completely off the stellar disc:

$$
\begin{aligned}
F(v, t)= & F_{0}(v)-\sum_{n} \sum_{i, j}^{\text {ring }_{n}} f_{i j}(v)\left(1-\mathrm{e}^{-\tau_{n}}\right)\left(1-u_{1}\left(1-\mu_{i j}\right)\right. \\
& \left.-u_{2}\left(1-\mu_{i j}\right)^{2}\right) .
\end{aligned}
$$

\section{THE DEPENDENCY OF THE LINE PROFILES ON THE RING PROPERTIES}

In the previous section we presented our model for simulating the expected line profiles resulting from a transiting planet plus ring system. In this section we investigate the impact that different parameters have on the observed line profiles. For these simulations 

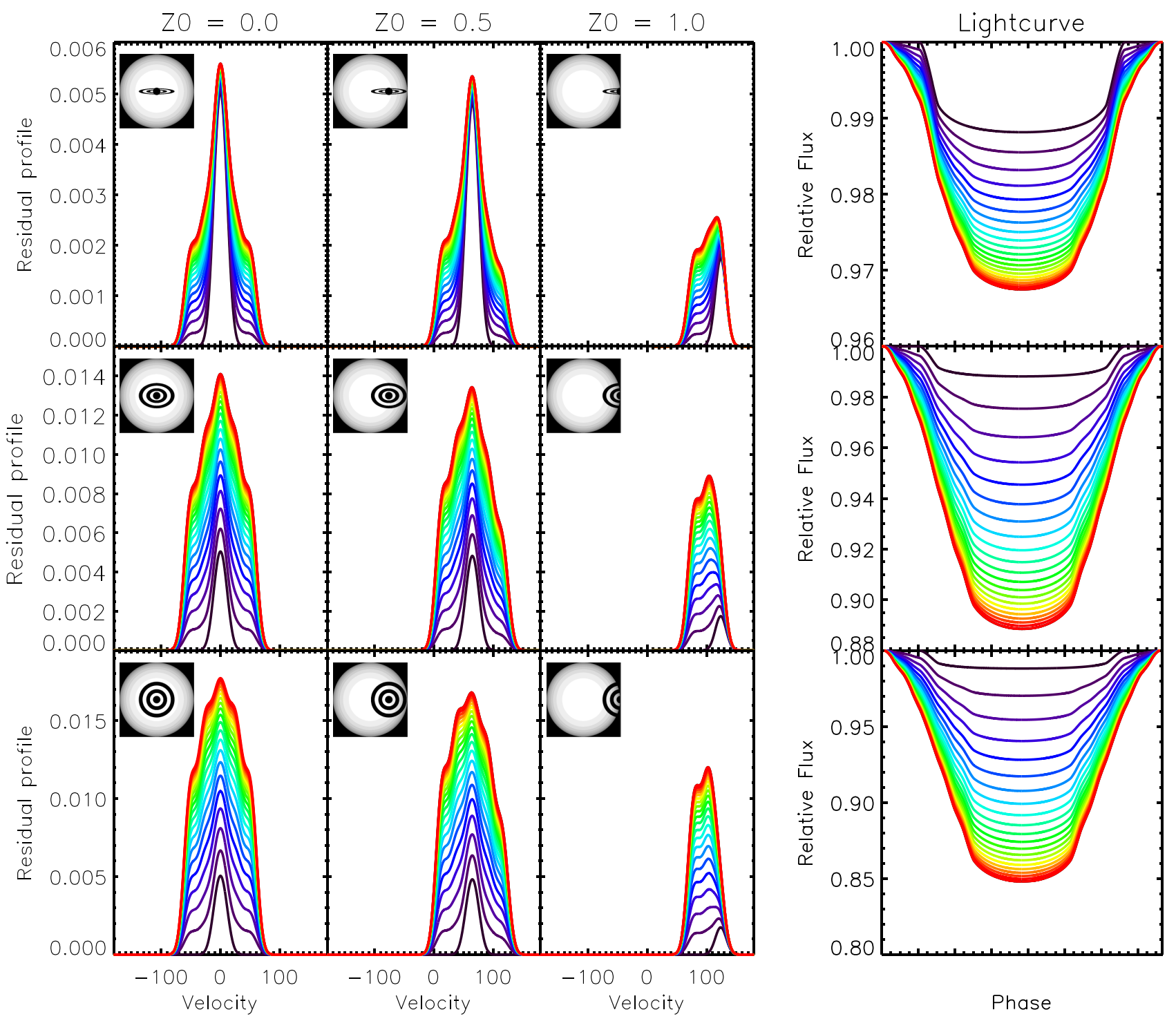

Figure 1. Illustration of the impact of the obliquity of the rings on the observed residual line profiles (left three panels) and light curve (right panels). The top row is for an obliquity of $80^{\circ}$, the middle row for an obliquity of $45^{\circ}$ and the bottom row for an obliquity of 0 deg. The residual line profiles are plotted for different distances from mid-transit, $Z_{0}$, given in units of the stellar radius. Note that, for clarity, we have removed the offset between the individual residual profiles. The colour scale for both the light curves and residual line profiles shows the effect of increasing opacity, $\tau$ (black to red: $\tau=0$ to $\tau=2.5$ ). For these simulations the position angle on the sky was set to $\mathrm{PA}=90^{\circ}$, the impact parameter $b=0$, and the intrinsic line profile has a FWHM $\gamma=20 \mathrm{~km} \mathrm{~s}^{-1}$.

we set the stellar parameters to approximate those of $\beta$ Pic. We assume a stellar rotation of $v \sin i=130 \mathrm{~km} \mathrm{~s}^{-1}$, an intrinsic line width of $\gamma=20 \mathrm{~km} \mathrm{~s}^{-1}$ full width at half-maximum (FWHM), and $V$-band limb darkening coefficients for an effective temperature of $8000 \mathrm{~K}$ and $\log g=4.0$ from Claret (2000).

We set the planet-to-star radius ratio to $R_{\mathrm{p}} / R_{\star}=0.1$ (comparable to the radius ratio derived from the radius measurement for $\beta$ Pic b from Currie et al. 2013), and consider a simple ring system with two rings that have the same opacity, $\tau$. We vary the optical depth from 0 to 2.5 in steps of 0.125 (note that for Saturn the optical depth varies from $\sim 0.05$ to $\gtrsim 5$; e.g. Colwell et al. 2009; Hedman \& Nicholson 2016). The inner ring starts at $1.8 R_{\mathrm{p}}$ and extends to 2.8 $R_{\mathrm{p}}$ and the outer ring starts at $3.8 R_{\mathrm{p}}$ and extends to $4.8 R_{\mathrm{p}}$. We note that the rings modelled here are larger than Saturn's rings, which extend from $\sim 1.2$ to $2.3 R_{\text {Saturn }}($ Cox 2000 ), as we are considering more massive ( $\beta$ Pic b like) planets. To show the effects of altering the ring parameters more clearly, we also calculated residual line profiles, $F_{\text {res }}(v)$, with respect to the unocculted stellar profile, $F_{0}(v)$ (e.g. Cegla et al. 2016).

$F_{\text {res }}(v, t)=F(v, t)-F_{0}(v)$

We note that the correct normalization of $F(v, t)$ is very important, and requires a precise knowledge of the transit light curve in order to obtain these residual profiles. For real data, this may not always be possible; however, we show that the parameters of the ring system can still be recovered by fitting the full profiles (see Section 4).

In Figs 1 to 4 we show the impact of varying the obliquity of the rings, the impact parameter, $b$, the PA of the rings on the sky, and the width of the intrinsic line profile, $\gamma$. We also show the impact of these parameters on the transit light curve. A cursory inspection of the results presented in Figs 1 to 4 clearly shows that the presence of a ring system can have a significant impact on the line profiles - and their presence can be inferred from a single 'snapshot'. This is in contrast to the transit light curves, where the clearest signal 

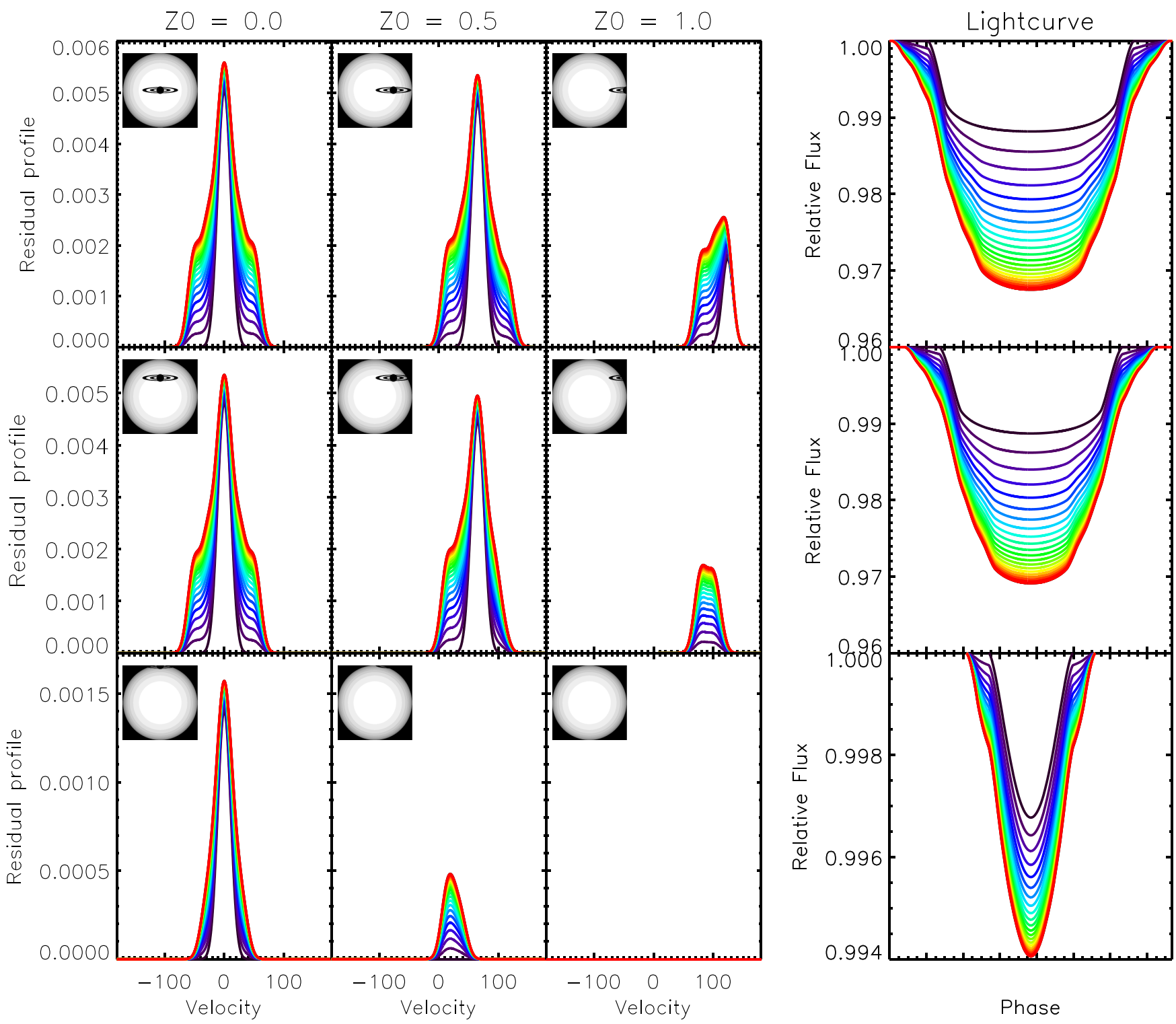

Figure 2. Same as Fig. 1, but now showing the effect of increasing the impact parameter, $b$. From top to bottom we show $b=0, b=0.5$ and $b=1.0$. For these simulations the rings were inclined by $80^{\circ}$ from face-on.

of the presence of a ring system occurs during ingress and egress requiring good data sampling at those times.

\subsection{Varying the ring parameters}

As can be seen from Fig. 1, for a nearly edge-on ring system, increasing the opacity does not have a significant impact on the centre of the line profile. This is as expected, since the rings mainly occult areas of the star that are offset in velocity from the planet's disc. In addition, the gap between the rings is visible as a flattening of the profile between 40 and $50 \mathrm{~km} \mathrm{~s}^{-1}$. This is in line with expectations for a star with $v \sin i=130 \mathrm{~km} \mathrm{~s}^{-1}$ and a ring gap between 0.28 and $0.38 R \star$, for which the projection of the ring gap corresponds to an offset from the centre of the line profile by $36 \mathrm{~km} \mathrm{~s}^{-1}$ (at the inner edge) to $49 \mathrm{~km} \mathrm{~s}^{-1}$ (at the outer edge).

As the planet moves along its orbit, the asymmetry in the profiles becomes more apparent. In the first instance this asymmetry is caused by the gradient of the limb-darkening across the stellar disc, while closer to egress the asymmetry is caused by the fact that only part of the planet and rings are occulting the star. When changing the obliquity towards a face-on ring system, the presence of two rings separated by a gap becomes more obvious as $\tau$ increases. This can be identified by the presence of the two 'shoulders' either side of the centre of the residual line profiles, and is most clearly seen at mid-transit.

The effect of changing the impact parameter $b$ is shown in Fig. 2. It is quite evident that, as expected, the duration of the transit decreases with increasing impact parameter. The effect on the residual line profiles is minor, except when the impact parameter is at 1 and only a small portion of the rings occults the star due to the grazing nature of the transit and nearly edge-on viewing angle of the rings in this particular simulation.

We show the impact of changing the PA on the sky in Fig. 3. When the rings are aligned perpendicular to the orbit of the planet, 

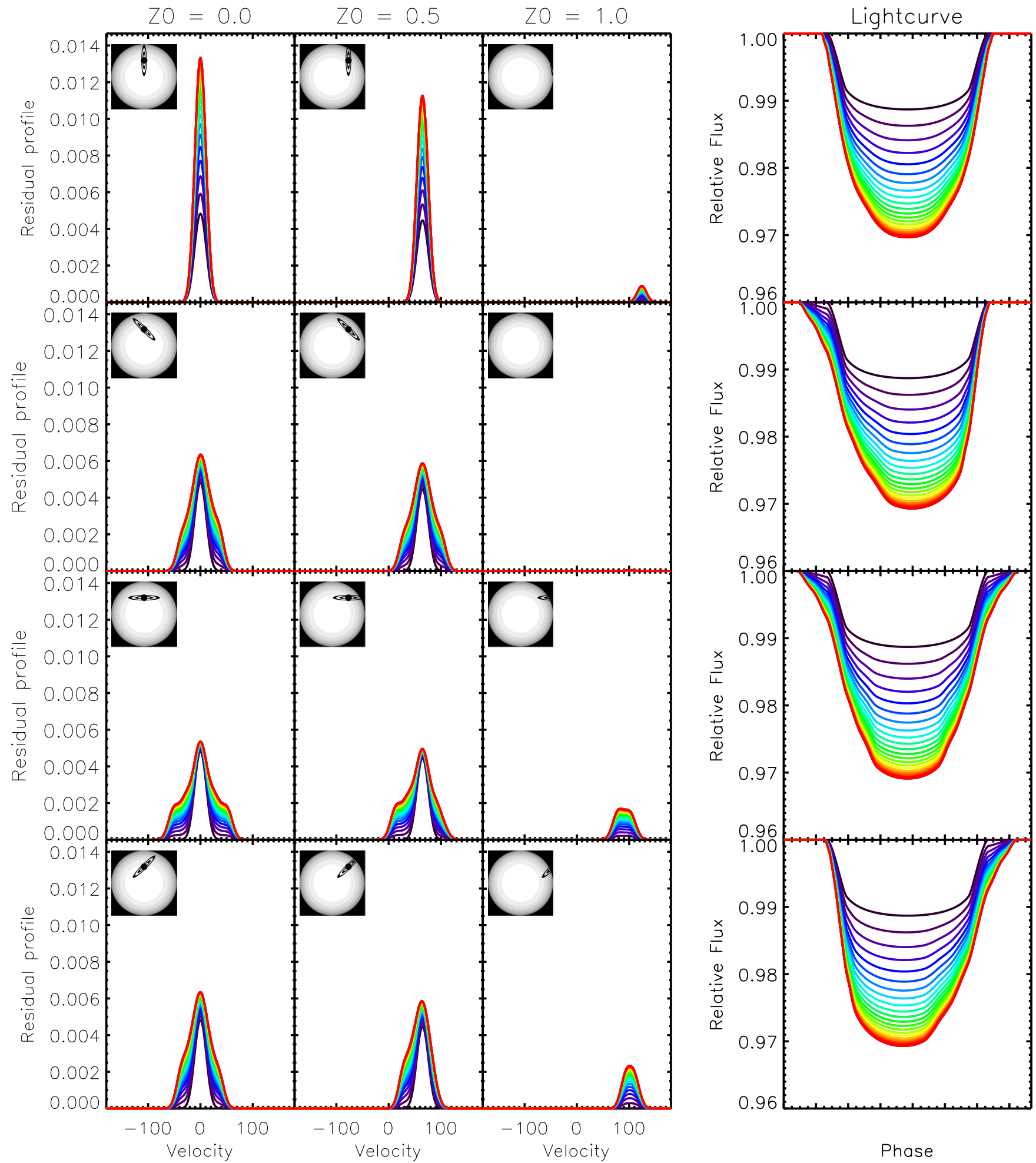

Figure 3. Same as Fig. 1, but now showing the effect of the PA of the rings. From top to bottom we show $\mathrm{PA}=0^{\circ}, \mathrm{PA}=45^{\circ}, \mathrm{PA}=90^{\circ}$ and $\mathrm{PA}=135^{\circ}$. For these simulations the rings were inclined by $80^{\circ}$ from face-on.

the residual line profile remains narrow, but the amplitude of the residual profile increases significantly with increasing $\tau$. However, since there is no velocity gradient along the major axis of the rings, the ring gap is not clearly detectable. When the rings are misaligned with the planet's orbit (e.g. for $\mathrm{PA}= \pm 45^{\circ}$ ), the transit light curve becomes highly asymmetric. The line profiles are also asymmetric, especially for $z_{0} \lesssim 0$ for $\mathrm{PA}>0$ and $z_{0} \gtrsim 0$ for $\mathrm{PA}<0$. For these PAs the rings occult parts of the star with very different surface 

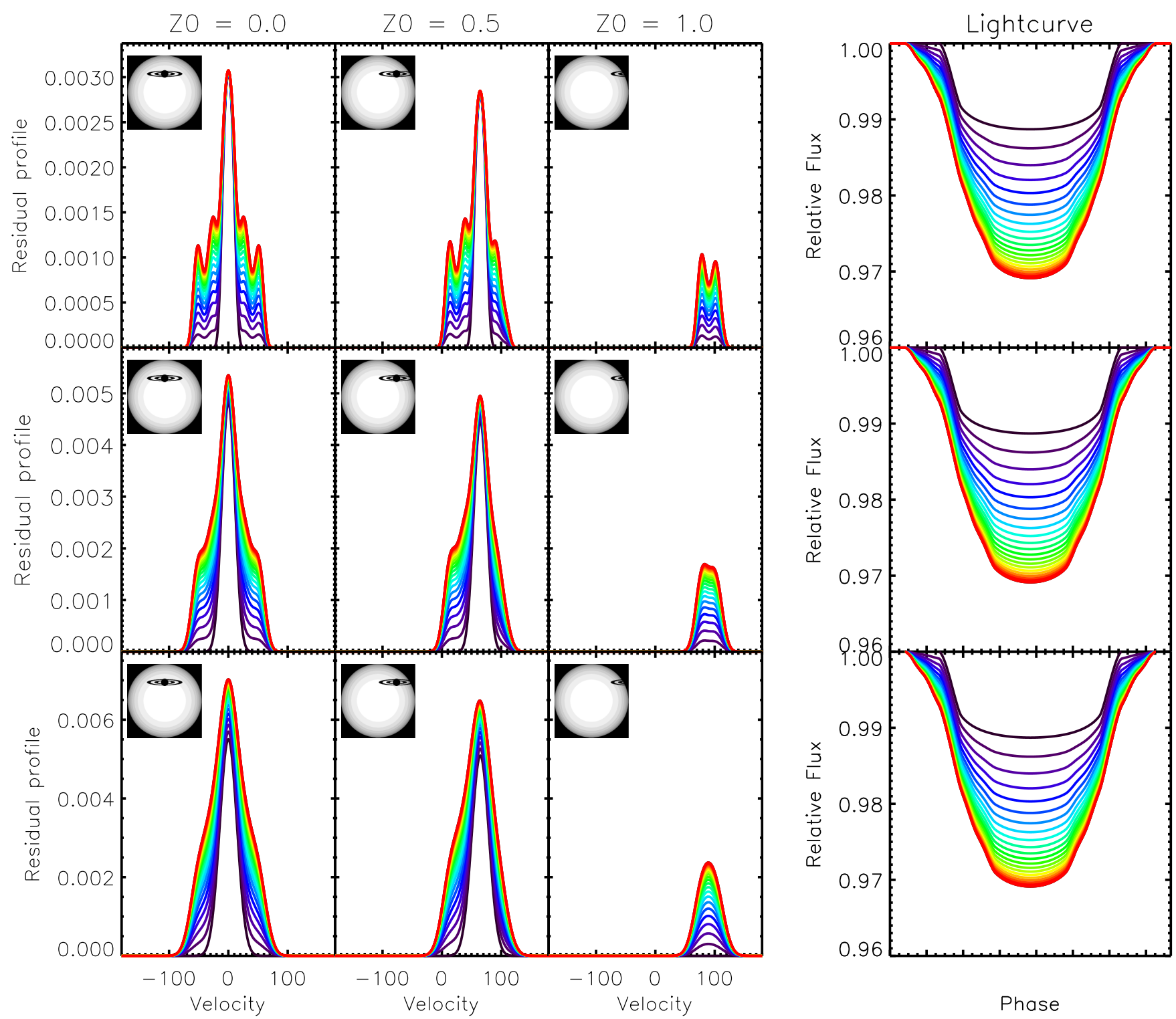

Figure 4. Same as Fig. 1, but now showing the effect of increasing the impact of the width of the intrinsic stellar line profile. From top to bottom we show the residual profiles for a FWHM of $\gamma=10 \mathrm{~km} \mathrm{~s}^{-1}, \gamma=20 \mathrm{~km} \mathrm{~s}^{-1}$ and $\gamma=30 \mathrm{~km} \mathrm{~s}^{-1}$. For these simulations the rings were inclined by $80^{\circ}$ from face-on.

brightnesses, while on the other side, the gradient in the limbdarkening from one end of the rings to the other is reduced.

\subsection{The effect of the intrinsic line-profile width}

The intrinsic line-profile width, $\gamma$, sets the fundamental limit that defines how well features can be resolved. In Fig. 4 we show the impact of changing the FWHM of the intrinsic line profile from 10 to $30 \mathrm{~km} \mathrm{~s}^{-1}$. It is clear that for a lower $\gamma$ it is easier to resolve features in the rings, and the two gaps can be clearly seen. However, as $\gamma$ is increased to $30 \mathrm{~km} \mathrm{~s}^{-1}$ it becomes harder to discern the presence of a clear ring gap. This implies that fast-rotating stars with a narrow intrinsic line profile are preferable for this type of observation, and that care should be taken with the selection of the wavelength region to be observed such that lines with a lower intrinsic broadening are targeted.

\section{RECOVERING THE PARAMETERS OF THE RINGS FROM SIMULATED OBSERVATIONS}

In the previous section we showed the impact of different parameters on the observed line-profile changes. However, since in reality it will be very difficult to obtain absolute spectra, getting direct measurements of line-profile variations will be difficult to achieve. It is therefore more useful to test how well the parameters can be recovered when attempting to fit the continuum normalized line profiles that are typical of high-resolution spectroscopic observations. To do this we generated a set of transit observations of different duration and with different properties of the rings to which we add noise, and that we subsequently fit using a Markov Chain Monte Carlo (MCMC) method.

To simulate the observations, we assume that the planet moves on a circular orbit, with a semi-major axis of 8.2 au and an orbital period of 18 years, similar to the short period case for $\beta$ Pic from 

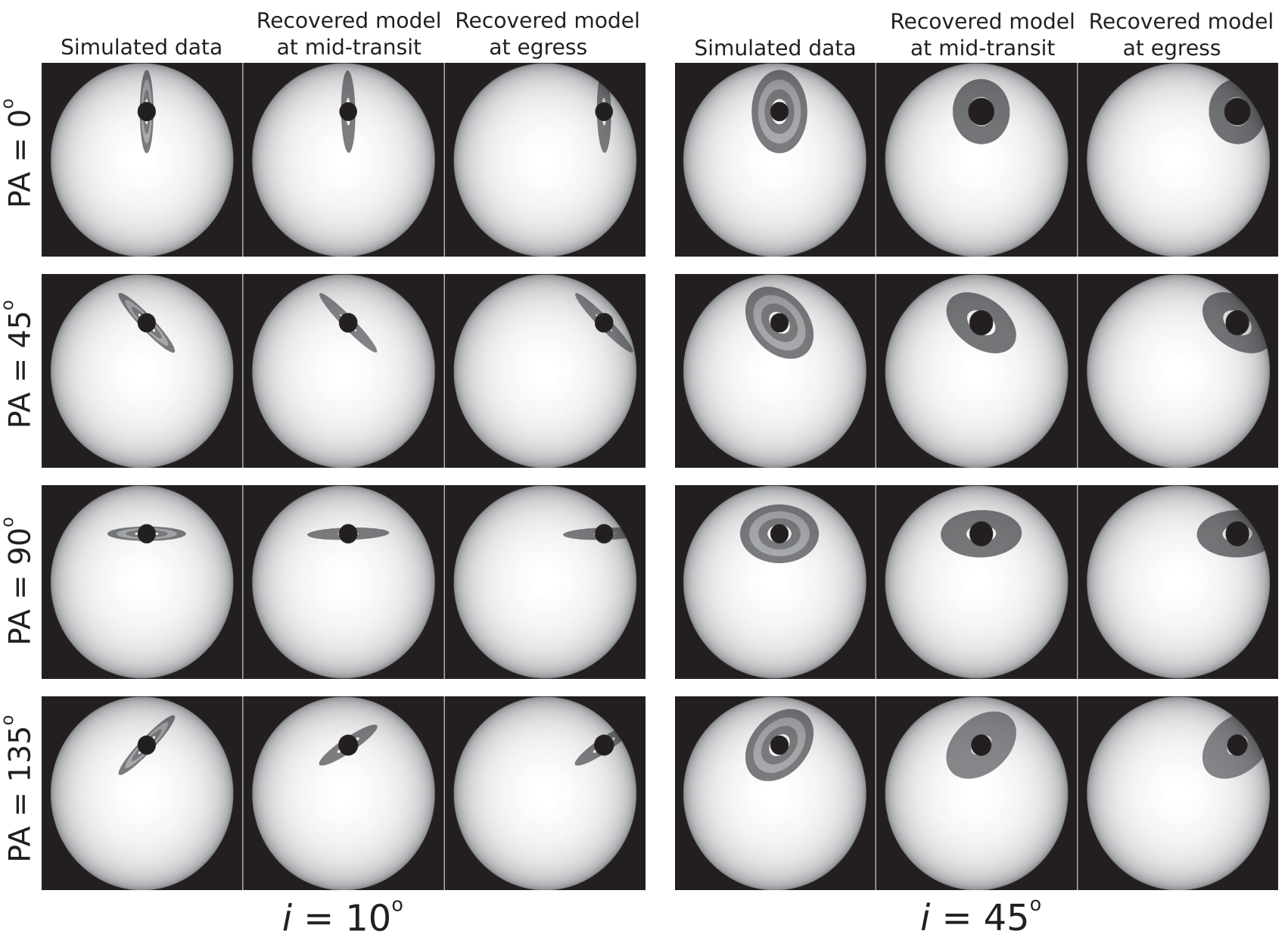

Figure 5. Examples of the simulated and recovered ring systems. For each set of three images, the left image shows the simulations just after mid-transit, the middle shows the best-fitting model at mid-transit and the right panel shows the best-fitting model close to egress. All the fits were done to the simulations covering $2.5 \mathrm{hr}$ of observing time (100 frames). The left columns show simulations for rings with an obliquity of $10^{\circ}$ from edge on, while the right column is for an obliquity of $45^{\circ}$. From top to bottom the simulations are for a PA of $0^{\circ}, 45^{\circ}, 90^{\circ}$ and $135^{\circ}$, respectively.

Lecavelier des Etangs \& Vidal-Madjar (2016). For these simulations we adopted an impact parameter of $b=0.5$. We have also taken into account the resolution of the instrument, assuming a value of $R \sim 100000$. Since our simulated model profiles are oversampled at a resolution of $250 \mathrm{~m} \mathrm{~s}^{-1}$, we first convolve the simulated line profiles with a Gaussian at the instrumental resolution, and then rebin the convolved profiles to a grid of $1 \mathrm{~km} \mathrm{~s}^{-1}$, which is approximately the sampling used by most $R \sim 100000$ spectrographs. We also assume that the noise in each pixel is Gaussian with $\sigma=0.0014$ (signal-tonoise ratio $\sim 700$ ), which, for a star of the brightness of $\beta$ Pic, should be achievable with a 1.5 min cadence when using a high-resolution Echelle spectrograph on an 8-m-class telescope.

For each set of planet and ring parameters, we run two sets of simulations to test the impact of the timing of the observations on the ability to recover the properties of the ring. The first set is taken just after mid-transit, while the second set is taken midway through egress. For each of the two sets we simulate a single observation, a single block of 10 sequential observations (lasting $15 \mathrm{~min}$ in total), and finally a single block of 100 observations (lasting $2.5 \mathrm{~h}$ in total); again all exposures are assumed to be taken sequentially.

For the simulations we vary the PA between $0^{\circ}$ and $135^{\circ}$ in steps of $45^{\circ}$ for two different obliquities, $10^{\circ}$ from edge on and at $45^{\circ}$ from edge on. As before, we set $R_{\mathrm{p}} / R_{\star}=0.1$, but now simulate a slightly smaller system with three rings without gaps extending from 1.3 to $4.3 R_{\mathrm{p}}$. The first ring starts at $1.3 R_{\mathrm{p}}$ and ends at $2.3 R_{\mathrm{p}}$ and has an optical depth of $\tau=1$. The middle ring has $\tau=0.5$ and ends at $3.3 R_{\mathrm{p}}$. The final ring ends at $4.3 R_{\mathrm{p}}$ and has an optical depth of $\tau=1$.

After generating the individual simulations we fit each of them using a model with a single ring. To facilitate the fitting over a large parameter range we used a simple MCMC fit consisting of chains of 50000 steps.

\section{DISCUSSION}

The snap-shot simulations consisting of a single frame did not provide reasonable constraints on the recovered parameters, and we therefore focus on the simulations consisting of blocks of 10 and 100 frames (15 and $150 \mathrm{~min}$ ).

From our simulations and subsequent fits we find that, in general, we can obtain reasonable constraints on the main system parameters (obliquity, PA and outer ring radius). Fig. 5 shows a visual comparison between the simulations and the recovered models for the simulated data sets lasting $150 \mathrm{~min}$ (100 frames). By eye, it is 

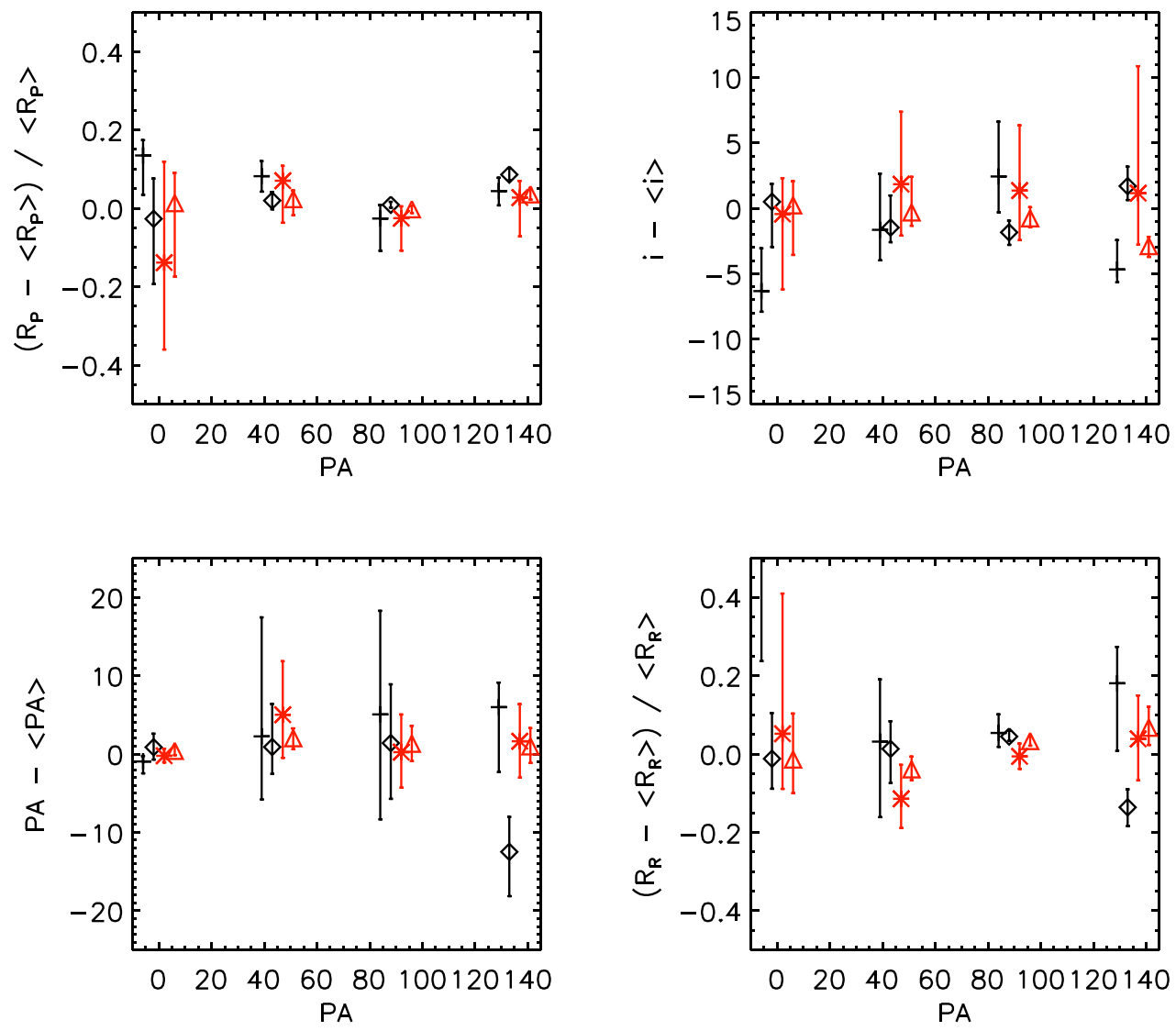

Figure 6. Differences between the recovered and original parameters for our simulations for an obliquity of $10^{\circ}$. Top left: relative difference in the radius of the planet $\left(R_{\mathrm{p}}\right)$. Top right: difference in the recovered obliquity of the rings $(i)$. Bottom left: difference in the PA of the rings. Bottom right: relative difference in the radius of the rings $\left(R_{\mathrm{R}}\right)$. The black points show the results for simulated observations near mid-transit, while the red points show the results for the simulated observations near egress. The plus and star symbols are for $15 \mathrm{~min}$ of observations (10 frames), while open diamonds and triangles are for observations lasting $150 \mathrm{~min}$ (100 frames). Note that the points have been offset in PA for clarity.

already quite clear that in most cases we can obtain useful constraints on the size, PA and obliquity of the ring system. The exception is for an obliquity of $45^{\circ}$ and a PA of $0^{\circ}$ (top right images in Fig. 5), where the recovered parameters result in a ring that is clearly more compact and circular. We attribute this to the fact that at an obliquity of $45^{\circ}$ the rings are already more circular and, to complicate matters further, at a PA of $0^{\circ}$ the projected major axis of the rings is aligned with the stellar rotation axis, leading to a degeneracy between the opacity and extent of the rings parallel to the projected stellar rotation axis.

From the images in Fig. 5 it is also clear that we are not able to fit the interior gap properly. This is not surprising, as in our input models the gap is only $0.03 R \star$ wide. This corresponds to an extent in velocity of $\Delta v \sim 4 \mathrm{~km} \mathrm{~s}^{-1}$, approximately four times lower than the intrinsic resolution of the line profiles. When fitting, it is therefore possible to make a trade-off between the size of the gap (location of the inner edge of the ring) and the radius of the planet.

A more qualitative analysis of our ability to recover the ring parameters is presented in Figs 6 and 7, where we show the fractional differences between the input parameters and the best-fitting parameters from our MCMC analysis. Again, it is clear that we can recover most parameters quite well. The exception is at $\mathrm{PA}=0^{\circ}$, when the major-axis of the rings is parallel to the projected rotation axis of the star, and the rings occult a very limited range in projected rotational velocities across the stellar disc. For an obliquity of $45^{\circ}$
(Fig. 7), the parameters are less well constrained than for the case of an obliquity of $10^{\circ}$. This can be understood by the fact that for an increased obliquity the rings appear more symmetrical, and changes in the orientation of the rings have a relatively smaller impact on the distortions in the stellar line profile.

Our simulations indicate that we can recover several fundamental parameters of the ring system, including the outer radius of the rings, the PA of the disc and the obliquity of the rings, without the need to observe the entire transit. This opens up the possibility to study ring systems for long period planets, where the transit duration exceeds the length of a single night. As expected, the simulations for 100 frames $(2.5 \mathrm{~h}$ of observation) provide a more robust constraint on the parameters than the simulations for $15 \mathrm{~min}$ of observations.

\section{CONCLUSIONS}

Using a simple model, we have shown that the added dimension of the stellar rotation allows us to directly determine the properties of rings around exoplanets that transit fast-rotating stars. We have also shown that this type of observation does not require us to observe the entire transit, making them particularly useful for planets at large orbital separations. However, we note that for higher obliquities, the properties of the ring system are less well constrained. This is due to the fact that for these systems the rings appear more symmetrical. Furthermore, for rings that have their projected semi-major 

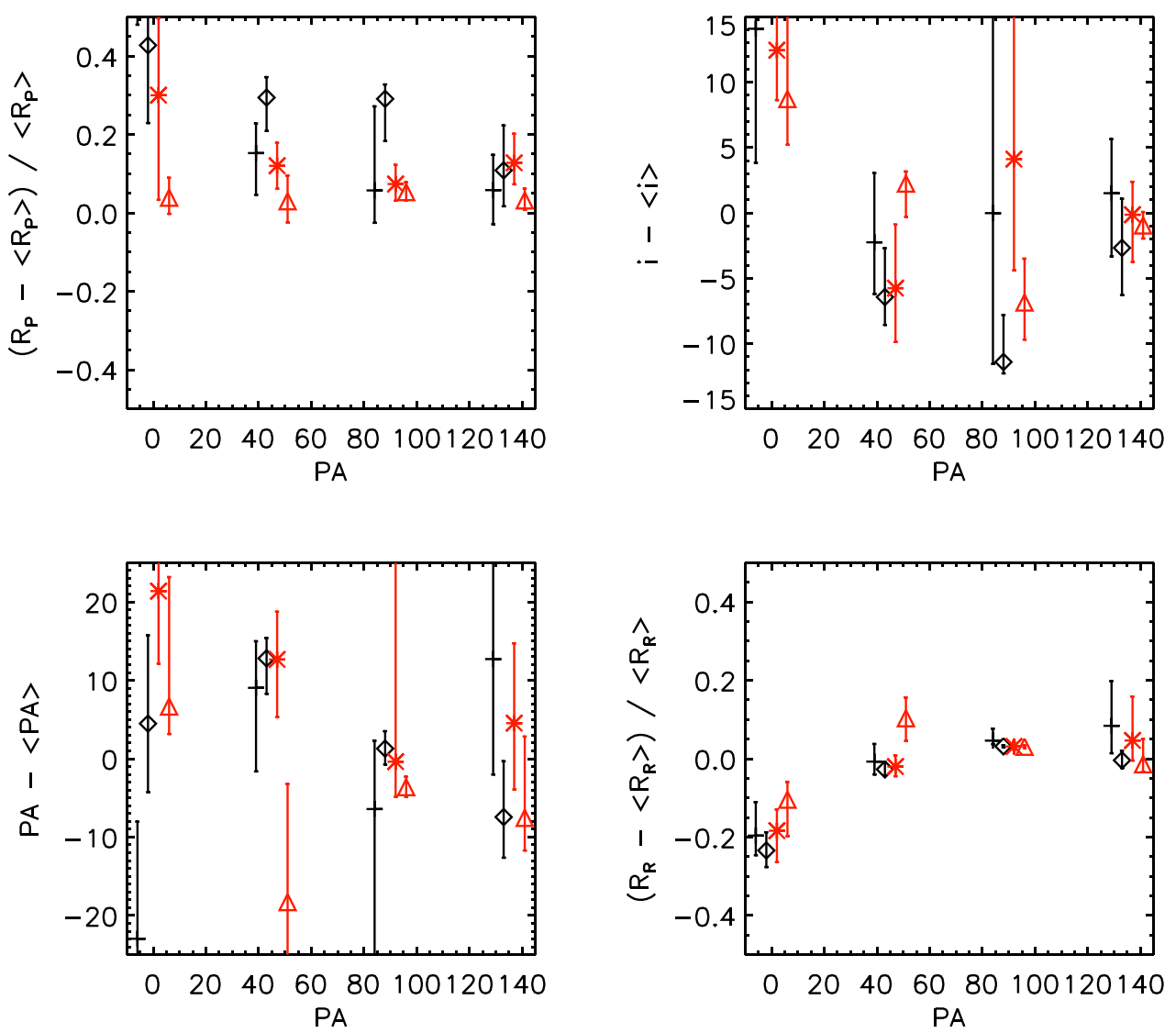

Figure 7. Same as for Fig. 6, but now for an obliquity of $45^{\circ}$.

axis parallel to the stellar axis of rotation, the reduced amount of velocities covered by the planet results reduces the constraints on the properties of the rings. We have shown the impact of different ring parameters on the distortion of the line profile, and demonstrated that the effects of individual rings become more obvious for narrower intrinsic line profiles.

\section{ACKNOWLEDGEMENTS}

EdM was in part funded by the Michael West Fellowship. CAW acknowledges support by STFC grant ST/P000312/1.

\section{REFERENCES}

Braga-Ribas F. et al., 2014, Nature, 508, 72

Cegla H. M., Lovis C., Bourrier V., Beeck B., Watson C. A., Pepe F., 2016, A\&A, 588, A127

Charnoz S., 2009, Icarus, 201, 191

Charnoz S., Morbidelli A., Dones L., Salmon J., 2009, Icarus, 199, 413

Charnoz S., Salmon J., Crida A., 2010, Nature, 465, 752

Charnoz S., Canup R. M., Crida A., Dones L., 2017, preprint (arXiv: 1703.09741)

Chauvin G. et al., 2012, A\&A, 542, A41

Claret A., 2000, A\&A, 363, 1081

Colwell J. E., Nicholson P. D., Tiscareno M. S., Murray C. D., French R. G., Marouf E. A., 2009, The Structure of Saturn's Rings. Springer, p. 375

Cox A. N., 2000, Allen's Astrophysical Quantities. Springer

Currie T., Thalmann C., Matsumura S., Madhusudhan N., Burrows A., Kuchner M., 2011, ApJ, 736, L33

Currie T. et al., 2013, ApJ, 776, 15
Dones L., 1991, Icarus, 92, 194

Fabrycky D. C., Winn J. N., 2009, ApJ, 696, 1230

Giles P. M., 2000, PhD thesis, Stanford University

Greaves J. S. et al., 2014, MNRAS, 438, L31

Harris A. W., 1984, in Greenberg R., Brahic A., eds, IAU Colloq. 75: Planetary Rings. Univ. Arizona Press, p. 641

Hedman M. M., Nicholson P. D., 2016, Icarus, 279, 109

Ip W.-H., 2006, Geophys. Res. Lett., 33, L16203

Kenworthy M., 2017, Nat. Astron., 1, 0099

Kenworthy M. A., Mamajek E. E., 2015, ApJ, 800, 126

Lecavelier Des Etangs A., Vidal-Madjar A., 2009, A\&A, 497, 557

Lecavelier des Etangs A., Vidal-Madjar A., 2016, A\&A, 588, A60

Lecavelier Des Etangs A., Deleuil M., Vidal-Madjar A., Ferlet R., Nitschelm C., Nicolet B., Lagrange-Henri A. M., 1995, A\&A, 299, 557

Mamajek E. E., Quillen A. C., Pecaut M. J., Moolekamp F., Scott E. L., Kenworthy M. A., Collier Cameron A., Parley N. R., 2012, AJ, 143, 72 Mazeh T., Perets H. B., McQuillan A., Goldstein E. S., 2015, ApJ, 801, 3 Millar-Blanchaer M. A. et al., 2015, ApJ, 811, 18

Ohta Y., Taruya A., Suto Y., 2009, ApJ, 690, 1

Pollack J. B., 1975, Space Sci. Rev., 18, 3

Roche E., 1849, Mémoire de la section des sciences, Académie des sciences et des lettres de Montpellier, 1, 243

Wang J. J. et al., 2016, AJ, 152, 97

Watson C. A., Littlefair S. P., Diamond C., Collier Cameron A., Fitzsimmons A., Simpson E., Moulds V., Pollacco D., 2011, MNRAS, 413, L71

This paper has been typeset from a $\mathrm{T}_{\mathrm{E}} \mathrm{X} / \mathrm{LT} \mathrm{E} \mathrm{X}$ file prepared by the author. 